\title{
CogITS: Cognition-enabled network management for 5G V2X Communication
}

ISSN 1751-8644

doi:10.1049/iet-its.2019.0111

0000000000

www.ietdl.org

\author{
Michael Taynnan Barros ${ }^{1}$, Gorka Velez $^{2 *}$, Harbil Arregui ${ }^{2 *}$, Estíbaliz Loyo ${ }^{2 *}$, Kanika Sharma1 , Andoni \\ Mujika $^{2 *}$, and Brendan Jennings ${ }^{1}$ \\ ${ }^{1}$ Telecommunication Software and Systems Group, Waterford Institute of Technology, Ireland \\ ${ }^{2}$ Vicomtech, San Sebastian, Spain \\ *E-mail:mbarros@tssg.org
}

\begin{abstract}
The $5 \mathrm{G}$ promise for ubiquitous communications is expected to be a key enabler for transportation efficiency. However, the consequent increase of both data payload and number of users derived from new Intelligent Transport Systems makes network management even more challenging; an ideal network management will need to be capable of self-managing fast moving nodes that sit in the $5 \mathrm{G}$ data plane. Platooning applications, for instance, need a highly flexible and high efficient infrastructure for optimal road capacity. Network management solutions have, then, to accommodate more intelligence in its decision-making process due to the network complexity of ITS. This paper proposes this envisioned architecture namely Cognition-enabled network management for 5G V2X Communication (CogITS). It is empowered by machine learning to dynamically allocate resources in the network based on traffic prediction and adaptable physical layer settings. Preliminary proof-of-concept validation results, in a platooning scenario, show that the proposed architecture can improve the overall network latency over time with a minimum increase of control message traffic.
\end{abstract}

\section{Introduction}

Transport is historically the critical driver to society's progress for providing degrees of efficiency in human locomotion. Future Intelligent Transport Systems (ITS) will contain applications and infrastructure that are going to revolutionise transportation as a whole and change people's life completely. It will be an entirely connected system of systems relying on cloud-based computational methods to enhance existing arduous tasks or to provide novel services including, e.g., traffic engineering, mobility-as-a-service, smart mobility, live vehicle monitoring, autonomous driving and driver assistance. Vehicle manufacturers already have the capabilities of processing large volumes of data from in-car sensors with limited connectivity for adaptive cruise control and assisted driving applications [1].

The main challenge ahead in this scenario (Fig. 1) is twofold, (1) how to develop a reliable ITS communication network infrastructure that has been promised for many years and yet not delivered, and (2) how to monitor this information remotely, or how to deploy the concept of live vehicles, in other words, the in-car real-time monitoring. These challenges will require that the network nodes (vehicles) are efficiently managed for constant data transfer regardless of the network complexity [2]. The problem of controlling the network node resources and its relationship with the existing network traffic and infrastructure is still an ongoing research topic namely, network management. With the increase of network complexity, such as in the ITS network, new methods must emerge with the goal of optimising network performance with minimum cost to network planning and operation [3]. The success of this action in ITS networks depends on the novel technologies proposed for the next network generation, the $5 \mathrm{G}[4]$.

The envisioned $5 \mathrm{G}$ sits on top of a heterogeneous radio access technology divided into two main parts, Fig 2. 1) the data plane composes of multiple techniques for improved data rate possible with higher frequency transmission and increased spectrum efficiency, 2) the control plane is a cloud-based management system that incorporates the existing autonomous network management solution for mobile networks. The control plane orchestrates the configuration of devices in the data plane. The self-management and orchestration can integrate the network as a plug-and-play-based architecture, through the softwarization of the network [5]. Since the

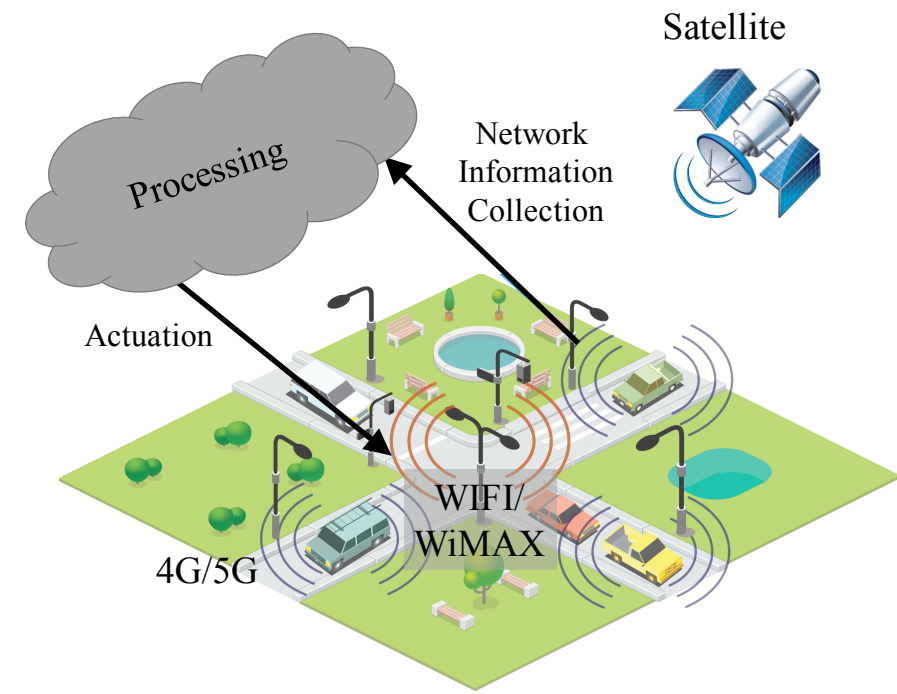

Fig. 1: Connected ITS scenario with a heterogeneous radio access technology.

next generation of mobile networks is a combination of communication and computation, collaboratively working in synchronisation supporting ultra data rate, its success relies on the evolution of flexibility and programmability in all non-radio 5G network segments [3].

This paper proposes Cognition-enabled network management for 5G V2X Communication, namely CogITS. The high-level architectural design is developed using cognitive network management concepts. This is a tentative analysis of how this network management solution can both improve instantiated network services while decreasing latency. We apply and evaluate machine learning techniques, towards an improved approach for the existing decisionmaking logic, thanks to the data pattern techniques implemented inside the management solution. Thereafter, we identify and classify traffic, anomalies and failures a lot more precisely than has been 
done before [3]. However, our intention is to show that for V2X communications, management solutions have to also rely on mobility information, which is very challenging for existing management approaches and rely on further improvement on autonomous-based solutions, in other words, machine learning-based network management is much appealing for this type of scenario. In this way, CogITS has the novelty of allowing both performance and flexibility to coexist by analysing both traffic and mobility network data. In summary, our contributions are highlighted as follows:

- CogITS full architecture design: We provide a design of a cognition-based architecture that has four main blocks: information processing manager, decision making manager, policy/ontology manager and actuation manager. Machine-learning methods are implemented in the decision making manager, and represent the main novelty of our architecture. We also include a device management component for managing different segments of the network and increase control and data layers coupling. Lastly we also provide a context manager, which increases overall architecture performance by synthesising network information in a compelling format to decrease signalling control messages.

- Analysis of CogITS performance in a platooning scenario: We obtained results for latency and the average percentage of control messages for platooning application in a V2X communication scenario. We show that both resource allocation and management can significantly interfere with the network performance.

- Increased orchestration performance based on roadside service migration: The proposed management solution increased the efficiency of the network infrastructure orchestration by managing service migration among road-side units while maintaining acceptable levels of latency. Even though the presented work is high-level, our main goal is to disseminate the idea of cognitive network management and to show its benefits in ITS scenarios.

The rest of this paper is organised as follows: First, in Section 2 , relevant literature on the topic is reviewed. Section 3 presents the cognition-enabled network management for 5G V2X communication (CogITS) followed by Section 3.1 with its challenges and requirements and Section 3.2 with the proposed architecture. Section 4 presents the analysis of the CogITS with simulations set up that are presented in Section 4.1 and the results presented in Section 4.2. A discussion is presented in Section 5. Finally, Section 6 presents the conclusion and future research directions.

\section{Literature Review}

In ITS communications, the synchronisation of both data and control plane is a key part of the whole system that enables remote and dynamic configuration and orchestration of virtual and physical resources [2]. Cognitive network management for 5G is emerging as the proper solution for that issue. The novelty of this new solution is the usage of one, or multiple machine learning techniques and their relationship, to both classify and characterise the network traffic with the ultimate goal of shaping network's configuration [3]. This concept introduces the capability of adapting an entire system based on historical data, which means in $5 \mathrm{G}$, that the network will fully understand system variables and optimally adjust their values for achieving a superior network configuration. However, that is also not enough in ITS because cognitive network management power is limited to the knowledge of the network. Due to the high dynamic environment of communicating cars, this system needs to perform its decision making over a context, where both virtual and physical resources information are quickly obtained and processed, making this task very challenging [6].

Existing approaches for the next generation of V2X communication based on software-based networks present very limited robustness inside a high-level network management. Some works have tried to tie the mobility and traffic data with the network management at some level (i.e., at radio access [7]), but they fail to provide full architectural solutions for management functionalities. Ning et al [8] discuss the need of fog computing for real traffic management, which is similar to the presented device management component and the context manager, which is supposed to be deployed in the fog. However, their technique has the disadvantage to not be deployed with machine-learning algorithms and might be present difficulties to be implemented. Another example of fog-based approaches can be found in [9], and even though they present an interesting technique for device management component, it is not clear how the obtained data will benefit the network. Other approaches take a step back and suggest management from the edge like in $[10,11]$. Their main advantage is the detailed workflow that establishes good relationship between the control layer and the roadside local controllers. They have concerns on the scalability and mobility management issues with their architecture, which is one of the main advantages that we aim to provide with CogITS. This literature review shows that recent advancements in the state-of-the-art on management of $\mathrm{V} 2 \mathrm{X}$ communication still undermine the processing of the data from both traffic and mobility and neglect the context it has and the impact it has on the overall network performance.

\section{Cognition-enabled network management for $5 G$ V2X Communication}

The softwarization of control planes for future networks allows dynamic deployment of the network functions based on Network Function Virtualization (VNF) and Software-defined Networks (SDN) $[12,13]$. SDN has been appointed as the leading choice for network efficient network flexibility in recent years. It was unclear how this recent technology will be managed in ITS due to the high mobility levels of network nodes. SDN is one of the key technologies underlying smarter traffic engineering \& network slices, which are dedicated end-end network resource allocation, with direct benefits to ITS regarding mobility management. Different dedicated network set up can now be applied to different mobility patterns such as urban or rural. But managing everything using network operators can be extremely time consuming and costly. That is why autonomicity is another key player in $5 \mathrm{G}$. A total autonomous infrastructure can bring the performance required to provide low latency network management and not denigrating the performance of application or services. And lastly, using a cross-layer approach, knowledgebased radio resource management will optimise channel access by knowing its state information (see Figure 2). This concept is highly beneficial in ITS due to the very dedicated radio resources, and the many issues in using higher frequency band communication [14].

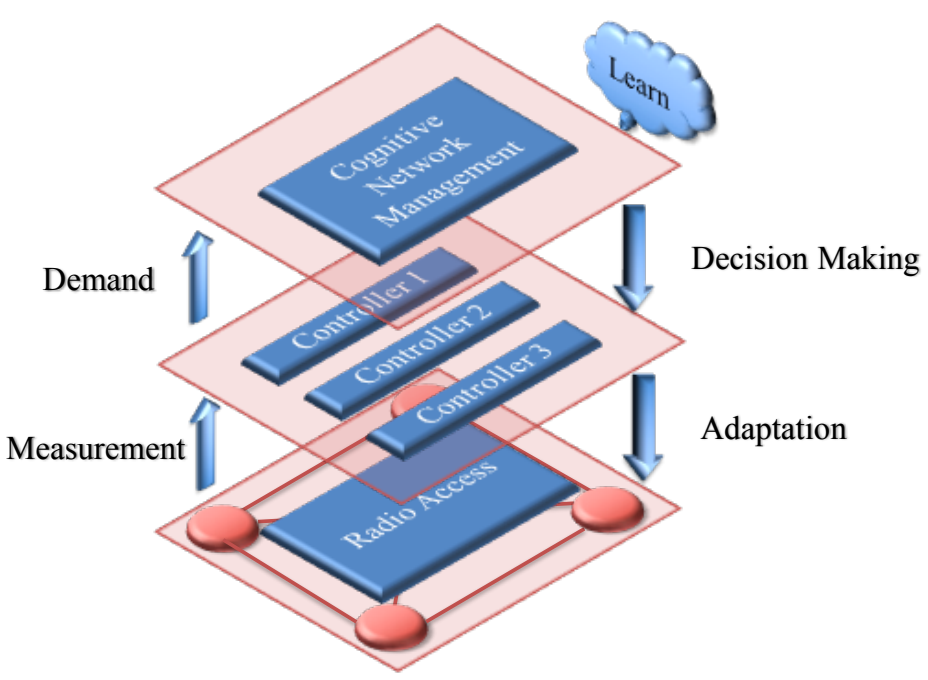

Fig. 2: Cross-layer network management solution, with the presented diagram, enables sharper network management and increase network performance due to near-optimum network configuration. 
Table 1 Mobility challenges vs communications needs

\begin{tabular}{l|cccc}
\hline & Scalability & Accessibility & Flexibility & Sustainability \\
\hline Mobility-as-a-service & High & High & Medium & Medium \\
Cooperative Systems & Medium & High & Medium & High \\
Smart Mobility & High & High & High & Medium \\
Transportation Planning & High & Low & Low & High \\
Autonomous Vehicles & High & High & High & Medium
\end{tabular}

The presented arguments for autonomicity in the network is aligned with the proposal of more intelligence in the network management [3]. Machine learning is then used to ensure the success of SDN, NFV, network slicing and resource management. It can be used to learn from historical data and make predictions or decisions, through management/creation of network policies and network orchestration eliciting. It can be used dynamically to adapt to new situations learning from new data, and in ITS; it can be used to learn specific mobility patterns that are in the roads, which can be highly useful for ITS application such as, smart mobility and mobility-as-a-service. Machine Learning is expected to be the key technology that will enable this vision of cognitive networking. However, the integration of machine learning to a $5 \mathrm{G}$ infrastructural architecture is still an open research question, due to the lack of quantification of the benefits of such technology. It is known that Machine Learning can be used for instance to forecast resource demand requirements or to detect security threats and error conditions. Nevertheless, there is still not any standard architecture to accommodate a machine learning module that enables a Cognitive Network Management.

\subsection{Challenges and requirements}

Mobility as a Service (MaaS) serves each user through a unique gateway with a combined private and public transportation applications. One example is Platooning, in which cars collaboratively self-organise to maximise road capacity. Moreover, many transportation resource sharing schemes have appeared inside the wide smart mobility concept, where not only vehicles (car sharing) but also trips (carpooling) are disseminated with the aim of sharing expenses as an electronic hitchhiking. That is where constant vehicle monitoring will be crucial. It will provide high-quality data for road managers and planners towards the transportation planning in urban and rural scenarios (e.g. custom Internet of Things (IoT) sensors, anonymous user traces or travel demand data from public transport smartcards), making this transportation planning on-demand very challenging. However, it could benefit society greatly, especially regarding safety and pollution.

Scalability and accessibility require high levels of communication for all applications in mobility. We are confident that mobility and communication systems must be integrated, transforming wireless networks to cope with the future ITS demands. Mobile communications are still pushing innovative research on tools and services that support instantaneous mobility decisions thanks to upto-date real-time information access for a wide variety of multimodal options and, at the same time, enabling technologies for data sensing regarding mobility supply and demand [15].

Many researchers are sceptic whether or not the next generation of mobile networks $(5 \mathrm{G})$ is going to be delivered on time to accommodate ITS needs and challenges. The network infrastructure has to handle the massive number of vehicles' connections and high data rate, supporting the autonomous cars remote control. Providing increased data rate while also providing efficient scalability actions is very challenging. We believe that the logical parts of ITS should move towards dynamically adaptable infrastructures. Routing, switching, and many other network functions should be customised by software only to accommodate specific properties of ITS.

Designing flexible infrastructures towards network resource optimisation is highly challenging and is in most case not possible. This is because the network complexity regarding topological and connection patterns is directly linked to its performance. In ITS, the complexity is further increased by their moving nodes and current management solutions for ITS lack controlled adaptable systems that handle such issues. Towards this direction, firstly, enabling infrastructure flexibility with moving nodes will rely on techniques that gather information about the actual state of the network. The network state means a set of variable that show the actual conditions of the network. Highly advanced estimation techniques are known to add complexity to the solution. Therefore, we see the role of machine learning more suitable for these above-mentioned challenges. Then, an efficient actuation solution must adapt the network taking into account the moving nodes while minimising both network control signalling and network performance interference, which can come from powerful policing and orchestration techniques.

\subsection{CogITS Architecture}

To demonstrate the benefits of the proposed paradigm, we designed a high-level heterogeneous and context-aware architecture that can manage the network by adapting its configuration based on the existing set-up of the network. It is defined under the superfluid principle, which allows the allocation of resources by a mobile-edge platform [16]. The architecture is termed CogITS and is introduced in Fig. 3. It is comprised of six major parts: Information Processing Manager, Decision Making Manager, Policy/Ontology Manager, Actuation Manager, Context Manager, and Device Manager.

1. The information processing manager: The information processing manager will retain data (data acquisition) from network managers or network controllers, network nodes and network context for storage (data storage) and formatting (data broker) according to the requirements of the decision making management. It will manage the dimensions and properties of the network data regarding integrity, longevity and update through the data broker. It can be accessed by a user interface, which allows management of the information by humans.

2. The decision-making manager: is the central part of the architecture. From the network data, it will create learning rules, predictions and scores, that will be incorporated in a reasoner. The reasoner is the highest level of cognition in that component, which will set up each type of machine learning to what context in what time this task will be executed. The machine learning module can be defined by the network manager as it pleases, with supervised learning methods, unsupervised learning methods or even reinforced learning. Typically, it is well documented the feasibility of supervised learning approaches for computer networks [17]. The reasoner is also responsible for validating the scores of both the prediction and the learning rules before going to the policy/ontology management. It also serves as a support of other major blocks, including the information processing management, the policy/ontology manager and the actuation manager. It is noteworthy that there is no pre-defined relationship in this block. The network manager can chose to have, with the reasoner, one or more of the other blocks in there, however, in this work we focus on the machine learning accompanied with a scorer, that prioritises the classes of traffic that are classified by the machine learning.

3. The Policy/ontologies manager: will, in broad terms, generate, optimise and distribute policies. Its manager will mostly create and update the policies, while its repository will store all of them. The repository will contain the policy continuum for policy classification and control and will update it according to the 


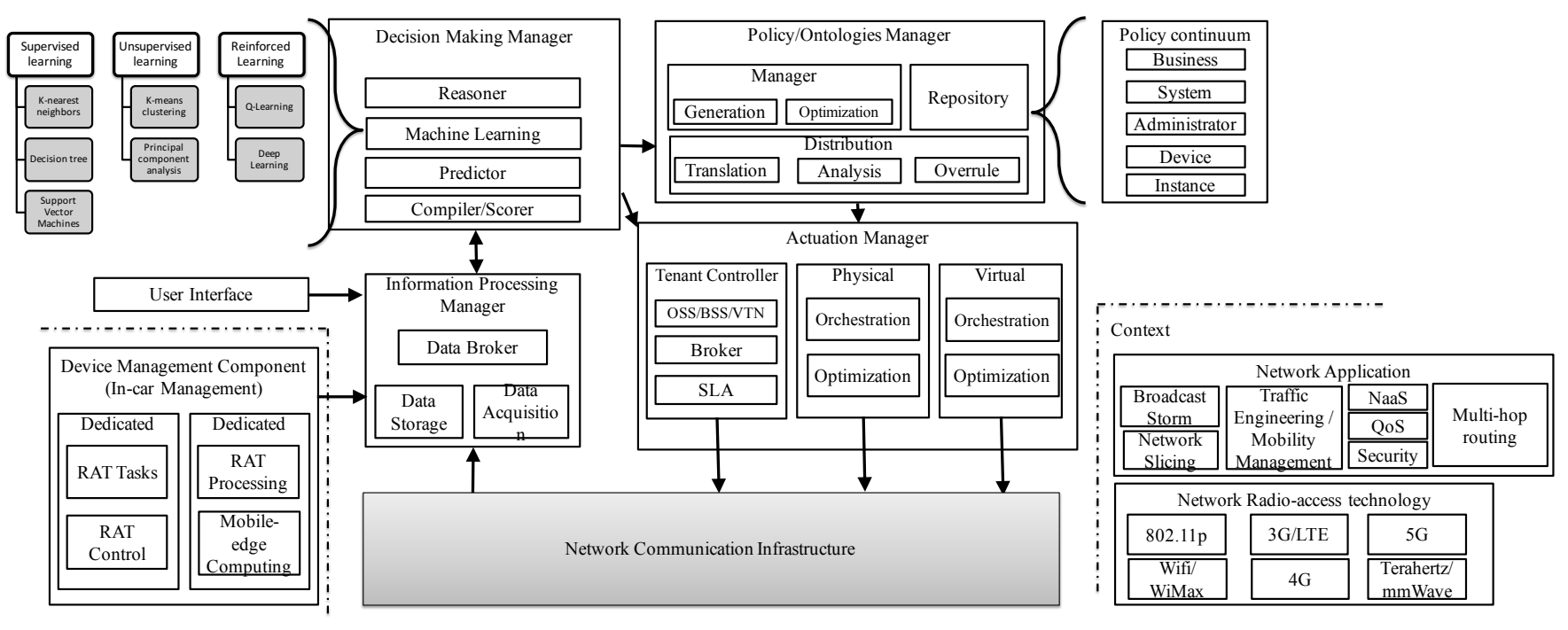

Fig. 3: The Cognition-enabled network management for 5G V2X Communication architecture (CogITS). It is comprised with six major parts: Information Processing Manager, Decision Making Manager, Policy/Ontology Manager, Actuation Manager, Context Manager, and Device Manager. It is also defined under the superfuild principle, which allows the allocation of resources with a mobile-edge platform.

results of the optimisation process, which includes the comparison between present and past performance scores. Lastly, the policies are distributed to the Actuation Manager, which if further processing and analysis is required, the distribution has the ability to provide further policy control.

4. The Actuation Manager: The distribution will work closely with the Actuation Manager. It will be responsible for making these policies be executed along both virtual and physical resources. The tenant controller will make sure the higher hierarchy of the network will also understand the policies. From the operational support system (OSS), business support system (BSS), and Virtual Network (VTN), the broker will analysed the internal policies to create a service layer agreement (SLA) with a network provider. Then the lower hierarchy can be re-updated with the policies. This process requires orchestration solutions that enable the many network nodes have updated policies.In the end, these controls are signalled to both physical and virtual resources by means of orchestration, which can be further optimised.

5. The Context Manager: The above management system is performed based on a pre-defined network context. This context is characterised by a set of a network application and a network radio-access technology (RAT). This information is necessary to 1) provide the proper set of policies from the policy continuum, 2) create network slices, 3 ) provide integration and heterogeneity with the existing infrastructure. This is important in ITS because an integrated effort in the radio-access technology is key to provide reliable connections in V2V and V2I settings by supporting different network radio-access technologies [18]. The proposed architecture can use the information about the connections to optimise them, while both channel characteristics, devices, and application are taken into account. In the network applications, our architecture can support context such as, broadcast storm, network slicing, traffic engineering, mobility management, Network as a Service (NaaS), Quality of Service (QoS), Security and multi-hop routing. In the networkradio access technology, our network management solutions can also take into account the type of connection between vehicles and the infrastrucuture, that have the following standards: $802.11 \mathrm{p}$, WIFI OR WiMax, 3G or LTE, 4G, 5G and Terahertz with mmWave future standards. The context will largely contribute to the policies regarding data rate and latency, which can be pre-defined here for each one of them.

6. The device management component: will be responsible for managing local node resources. It will provide the information processing manager with updated post-processed data while it is partially controlled by the actuation manager. In this way, the solution is robust enough to manage both micro and macro segments of the network. This is believed to be a key feature for future management solutions. Now, the network management can also manage the node's resources towards trade-off efficiency between performance and availability across the network. It will work closely with the radio access technology (RAT) for its tasks, control, processing and optional mobile-edge computing.

\section{Analysis of CogITS in a Platooning Scenario}

CogITS was designed to be the next step forward to flexible infrastructure by enabling live vehicles, online car monitoring, online passenger monitoring, micro-scale traffic engineering, platooning, etc. It is assumed then that complexity will be a challenge due to the increased average percentage of control messages affecting the efficiency of higher control levels of the network. However, the policy-based orchestration is a central part of the Cognitivebased network management paradigm, which can overcome such challenges. To initiate novel ideas in this direction, we present in this section a preliminary proof-of-concept for CogITS. We chose a platooning service initiation scenario that captures the benefits of machine learning and quick network actuation. In Platooning scenarios, cars are grouped into clusters where their cooperative effort maximises road capacity [19]. Therefore identifying and maintaining the infrastructure to accommodate these clusters is quite challenging [20]. It needs a robust network management infrastructure to provide not only cooperation among cars but also the in-car networking services. We aim to provide pieces of evidence of the feasibility of the CogITS while meeting a twofold criterion, which is latency and efficiency.

Figure 4 shows the proposed scenario that formalised the processes of resource allocation and service initiation of platooning application over the CogITS architecture in the form of a sequence diagram. The diagram shows eight entities including the vehicle user and the device management component, the radio access technology, local controllers, the information processing manager, the decision making and the policy/ontology managers, actuation manager and an application server. There are three different stages: 1) an end-end connection establishment, 2) resource allocation and 3) platooning initialisation.

The initial stage for connection establishment has to read the actuation manager for the network slice creation. In this way, the network 


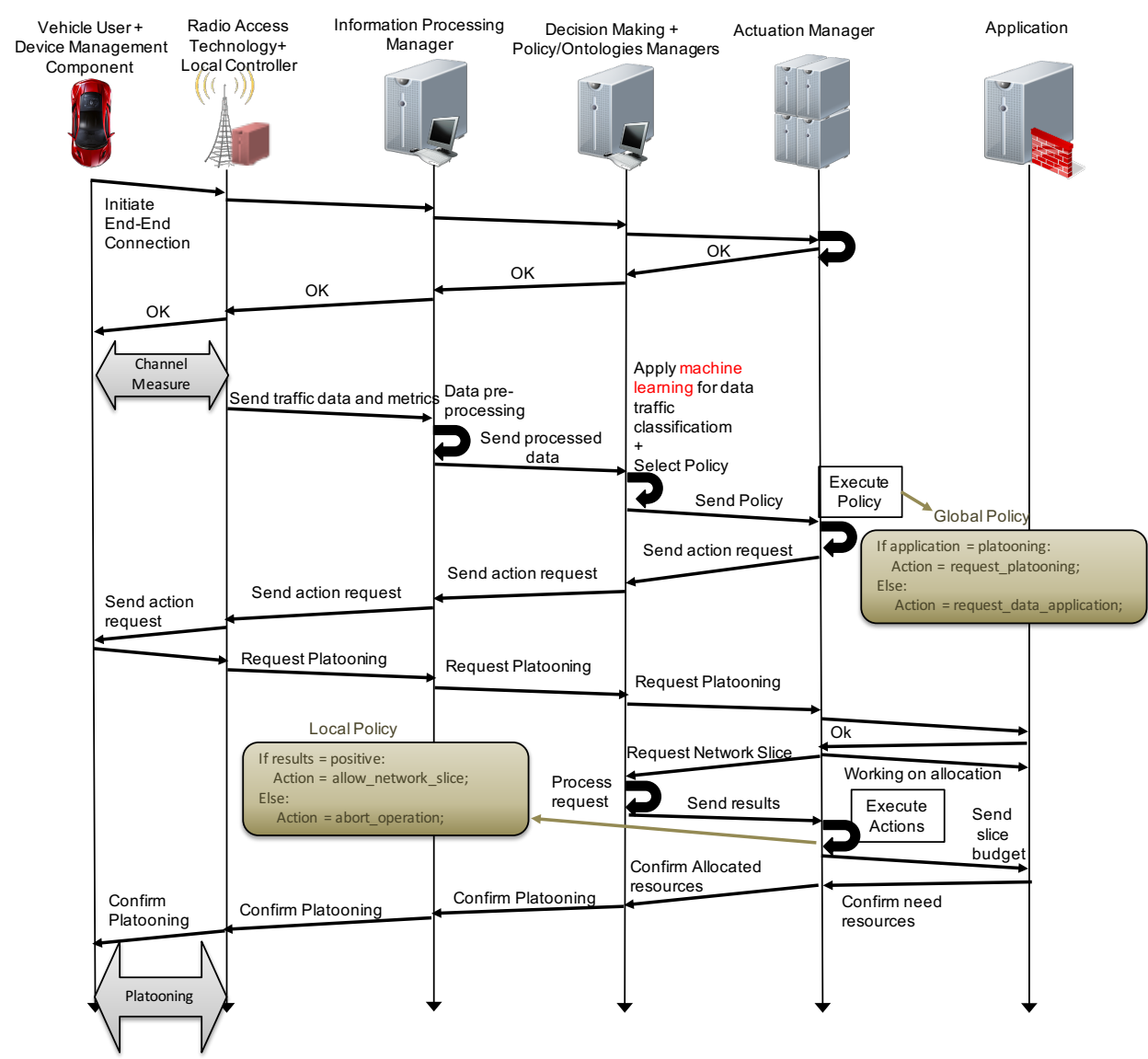

Fig. 4: Machine Learning managing a SDN/NFV-based network (CogITS approach).Platooning establishment-request sequence diagram as a proof-of-concept for the cognitive network management platform over a $5 \mathrm{G}$ infrastructure supporting ITS.

is capable of handling ITS communication requirements and establish the end-end link between V2X. Then, through the cross-layer cognitive network management, CogITS can verify the current state of the physical links, and adapt their performance based on the history profile built on that infrastructure as well as, the current state, future state, and service requirements. This has to be performed very quickly, and the challenge is into how to collect information to generate the best policing possible. Lastly the application service request, for platooning in this case, needs to be instantiated. The main part of the protocol the message exchange is between the CogITS, the actuation manager and the application server directly. Since the network slice is already instantiated, both decision making and actuation managers need to guarantee that the network slice is changed accordingly to accommodate resource-application requirements. Both the V2X connections states and the platooning scaling would be factors that interfere with the maintenance of the network slice over time. For example, policy changes can be made if applications are changed in the network nodes or infrastructure changes if the platooning clusters have increased or decreased. However, since CogITS deals with live data from the independent network nodes, this negotiation can be done independently as well.

\subsection{Simulations Set-up}

In platooning, the flows are based on geographical location of vehicles rather than its MAC/IP address. We manage network mobility by distributing vehicular connection flow tables into the available set of controllers in a given range of access points that the platoon falls into. To demonstrate the intricacies of this systems, we investigated two types of management arrangements for platooning. In the first, we used the CogITS to simulate the coordination among RSUs. This will demonstrate how the policy and orchestration can be performed. Three local controllers are coordinated by East/West-based orchestration interface and have three Roadside units (RSUs) each. The
Table 2 Simulation parameters and respective values for the platooning scenario.

\begin{tabular}{c|c}
\hline Simulation Parameters & Values \\
\hline Road Traffic Mobility Simulation & SUMO \\
Network Emulator & Mininet-WiFi \\
Number of local controller & 3 \\
Number of RSU & 6 \\
Road segment & $1000 \mathrm{x} 1000 \mathrm{~m}$ \\
Node density & 20 nodes \\
Wireless Interface for V2V & $802.11 \mathrm{p}$ \\
Transmission Range & $100 \mathrm{~m}$ \\
Velocity Range & $10-30 \mathrm{~m} / \mathrm{s}$ \\
Simulation Time & $200 \mathrm{~s}$ \\
Number of vehicle cluster & 1
\end{tabular}

Table 3 Machine Learning Accuracy Results

\begin{tabular}{l|c}
\hline Technique & Accuracy $(\%)$ \\
\hline Random Forest & 92.6086 \\
J48 & 86.2181 \\
Naive Bayes & 85.4188 \\
k-nearest & 92.6047 \\
Regular & 70 (aprox.)
\end{tabular}

decision-making manager will decide whether the platooning service data will use the first or the second solution based on the incoming data. This will be to its accordingly policies. Different types of machine learning can be incorporated into our scenario and similar ones as well, especially management systems based on centralised decision making. The cluster state is stored in the policy table in the Policy/Ontology manager. We assume ideal information processing 


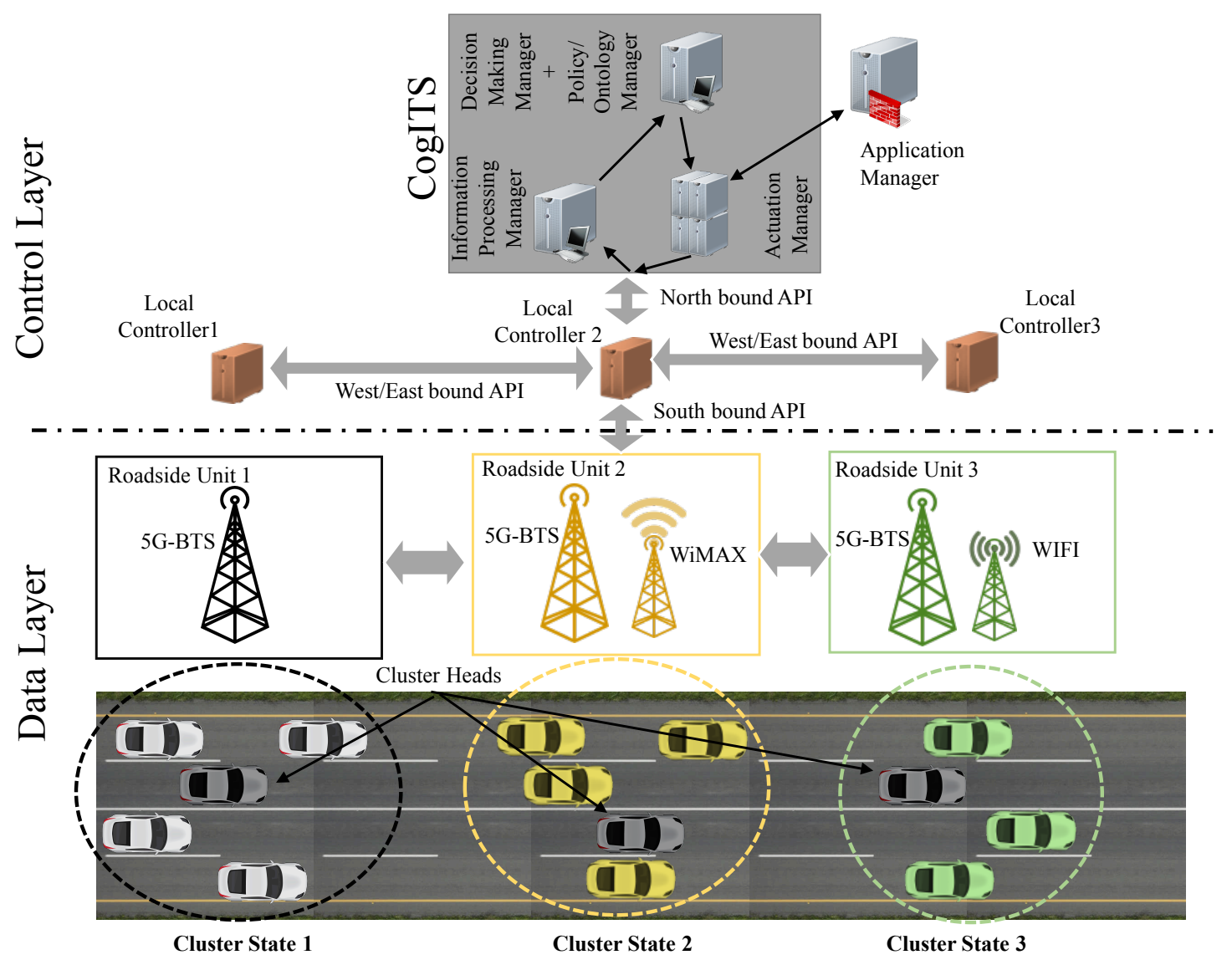

Fig. 5: Platooning scenario description following a centralised cloud-based architecture for the control plane. The CogITS solution is installed in the cloud whereas the data plan will be sited in the roadside unit platforms. This separation allows the flexibility necessary to perform high-level traffic data processing with machine learning without interfering with the network performance.

and pre-defined policies, which means that the network dynamics does not interfere in those blocks for now. We do not explore the complexity of the policy/ontologies managers due to simplicity. This interface with the actuation manager, which coordinates the controllers and also provides service migration. The continuity of a particular service and the overall stability of the network requires the vehicular flow table from the platoon maintenance by the local controller it is connected to. In this management approach, this flow table and the state of the service is transferred to the next controller when handover between controllers is performed, which is referred to as "service migration". This coordination scheme of controllers is essential in multiple domain SDN scenarios, with single or different service providers. In the second one, we used the conventional distributed network management. The three isolated local clusters are distributed and control 2 RSUs each. The management is simpler; the same policies are used however no service migration is performed. Our objective is to show how the platooning can be initiated and maintain acceptable levels of communication latency, even while more robust network management compared to regular network management is presented. The regular network management is characterised by a port-based traffic classification, a set of pre-defined policies based on the application port and a resource orchestrator without network slices.

We defined transmissions for both data and video streaming applications for V2X connections of the platoons. We monitor the latency and control overhead for the two cases network management approaches, the regular and CogITS. Following the CogITS architecture, the network performance that is processed, classified and decisions about the network for adjusting infrastructure is then made. We achieve the coordination of distributed controllers by enabling the SDN architecture in the Device Management Component. One node within the cluster acts as a control entity with the support of connected local controllers for network state management by issuing global policies. The coordination of distributed local controllers faces similar challenges like synchronisation without the increase in management overhead, like any other distributed system. In our model, the connectivity information of the platoon is stored in the policies of each local controller, the geographical location of the cluster head and the nodes inside a particular cluster. That information is migrated directly to the next controller to reduce the overhead in the control plane. The signalling between the architecture and the infrastructure, which are divided into control and data planes respectively, are made through the North, South and West/Eastbound APIs, Fig. 5. This is an approach based on the one proposed by Bagci et al. [21]. For coordination of controllers, the RSU's within each domain are inter-connected. By sending messages to RSU outside the given network domain (between planes), the Local Controller 2 is in our case discovered, which will propagate this agreement to Local Controllers 1 and 3. To send minimum information from one Local Controller to the next, the route to the Cluster Head is transferred to the next RSU, which forwards this information to Local Controller 2 and so on. The Cluster Head contains the overall topology and resource state of the cluster, meaning it is the direct responsible for cross-controller communication. In the case of isolated local controllers, the moving platoon needs to undergo the rediscover mechanism. We implemented this scenario in the Mininet-WiFi, which is an extension of Mininet emulator to support softwaredefined wireless network [22]. We needed the software-defined extension to be able to achieve the decoupling of data and control layers so that the cognitive management system can be re-trained if necessary as well as policies are updated on demand. This emulator is suitable for ITS research due to their Node-Car architecture which supports moving nodes that can be controlled by OpenFlow 
Controllers (via a switch). It supports vehicle-to-vehicle communication through a wireless mesh network and vehicle-to-infrastructure through a wireless interface. They also have integration with SUMO (Simulation of Urban Mobility) which is a road traffic simulator that implements our chosen platooning scenario. We provide more details about the simulations parameters in Table 2, which were chosen to create a borderline complex platooning configuration that needs a cognitive-based network management solution.

\subsection{Results}

We conducted a preliminary study on four machine learning techniques, including Random Forest, J48, Naive Bayes and IBk $(\mathrm{KNN}=1$, windowSize $=0)$ or simply k-nearest. For the performance evaluation of these machine learning techniques, we have collected packets from both data and video transmissions in the network controllers, Fig. 5. Both video and data transmissions use the TCP protocol, and the following features were measured at the packet level: timestamp, source id, destination id, protocol, packet length, packed id, time to live, source port and destination port. We have conducted preprocessing of the acquired data sets for redundancy elimination. We have observed, through Table 3, that Random Forest and k-nearest had $>90 \%$ accuracy, while the other performed below that threshold. These results are satisfactory compared with the regular approach for decision making, which is mostly based on port classifiers (performance around 70\%). These results are well documented in the area of IP traffic detection [17]. However, it is worthy to replicate for V2X scenarios.

The impact of the machine learning for both data and video average latency, Fig. 6 (a) and (b), show the variance of latency based on the individual technique performance. This shows how decision making has an impact on a single network measure. In average, machine learning techniques with accuracy $>90 \%$ performed better than the regular approach around $40 \%$ for data and around $10 \%$. These techniques can be further explored to other parts of the network ranging from the applications and network services. These capabilities have to be explored in more detail, specifically with the growth of encapsulated data traffic after the growth and interest in software-defined networks. For specifically ITS application, these machine learning techniques and policing can provide high levels of adaptation in the network. The overall latencies are still inside feasible range and can be worked on to be reduced, especially with the increase of control messages that maintain service performance, Fig. 6 . These results emphasise the categorisation of CogITS architecture into cognitive or distributed local controller on edge. Therefore, its learning capabilities can indeed make use of the data captured by the communicating vehicles to adjust better to the network conditions and achieve superior network performance through more efficient network management.

\section{Discussion}

Inside the literature review, we collected recent advancements in the state-of-the-art on management of V2X communication. As already mentioned, these works still undermine the processing of the data from both traffic and mobility and neglect the context it has and the impact it has on the overall network performance. Our proposed CogITS shows that it has potential to tackle this issues, however, we need more improved machine learning interactions with all managers to have an increased robustness level, and moreover, implement our approach in real test-beds for more impacting results. We believe that our work sets the initial work in this topic, however, more work need to be carried out specifically looking deeper at the problem of long-term mobility of cars that require interactions with the moving vehicles and updates in the machine learning models with a given frequency.

$5 \mathrm{G}$ requires a total change of the network infrastructure to accommodate software-defined networks and novel radio access technologies. The transition towards 5G infrastructure worldwide possibly will last 5-10 years. On top of that, $5 \mathrm{G}$ has to accommodate past mobile network generation for smoother transitions.

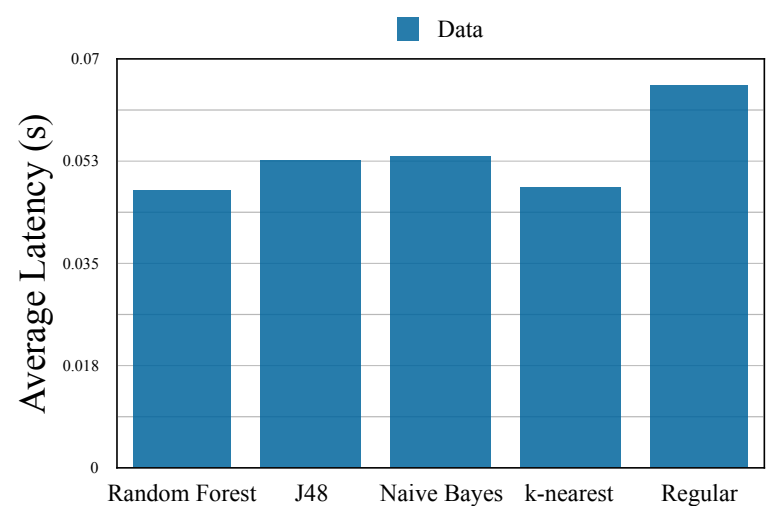

a)

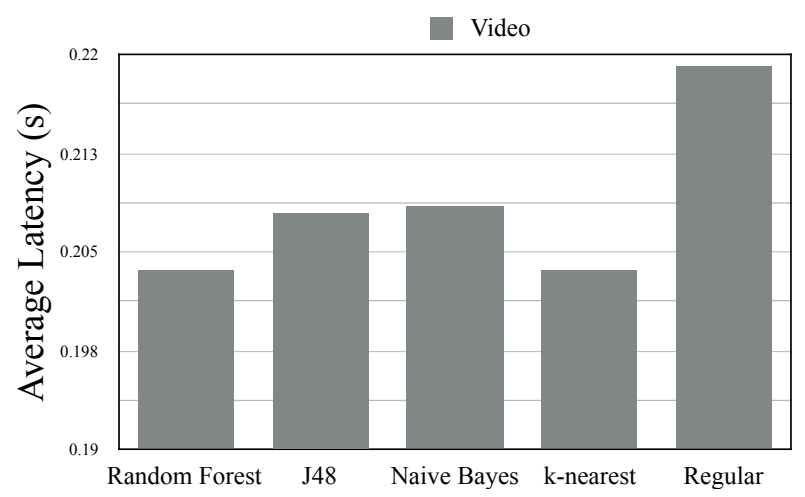

b)

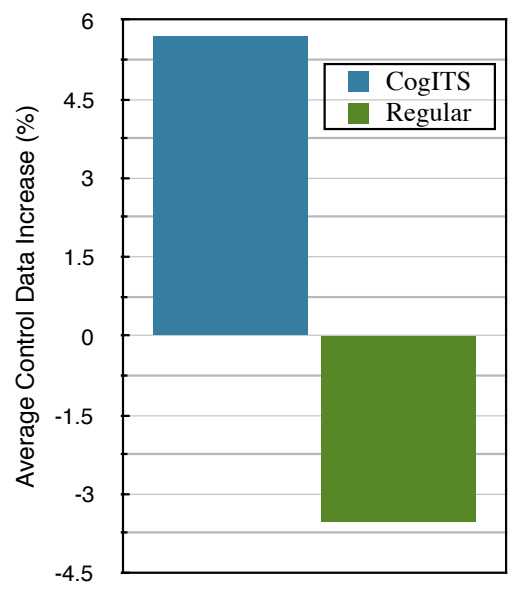

c)

Fig. 6: Impact of the machine learning performance on the average latency for data (a) and video (b) applications. (c) Percentage of control messages increase of both CogITS and regular management systems. These results emphasise on the categorisation of CogITS architecture into cognitive or distributed local controller on the edge by showing that the latency stabilises after a certain time due to its learning capabilities.

Such a challenging task will require a significant effort from both academia and industry in trying to simplify network operations despite growing technological diversity while proving availability for all communication technologies. 
Researchers envision 5G to being built on novel concepts towards existing and future infrastructure integration. We reckon that this novel solution must be developed based on the integration of SDN with software-defined radios (SDR), based on the presented management solution. With SDRs, software applications can implement $4 \mathrm{G} / 3 \mathrm{G}$ infrastructures or even older technologies. The SDR/SDN solution also provides integration with a cloud-based access technology, which enables the flexibility and reliability proposed for 5G. We finally can achieve interoperability between services and infrastructure with this proposed integration. The SDN/SDR integration can also be quite challenging because it requires a massive effort in changing the entire pre-existing infrastructure. The 5G-Crosshaul architecture was recently proposed recently, [23], for providing not only fronthaul and backhaul integration but also its full management and coordination. This solution will enable high capacity switches and heterogeneous transmission links for new radio access technologies managed by software-based solution in a multi-tenant and service-oriented approach. However, ITS requires a mobility-aware networking infrastructure with low latency performance, and it is not clear whether 5G-Crosshaul will reach this expectation, like the one presented regarding context-aware management. Likely, both fronthaul and backhaul have to be integrated with ITS data centre to support its related services, including mobility. The usage of the proposed architecture can solve this issue, by enabling the 5G-Crosshaul architecture with ITS network solutions. Such integration remains an open research topic of great importance to the success of $5 \mathrm{G}$ for ITS.

CogITS was designed to be the next step forward to flexible infrastructure by enabling envisioned application such as live vehicles, online car monitoring, online passenger monitoring, microscale traffic engineering, platooning and many others. It will require the integration of recent network advancements on top of heterogeneous radio access technology and robust network management [5]. And the main feature of CogITS that can perform all those tasks is the context-aware management. It can provide more easy integration in the performance spectrum, providing good mobile network endurance with a highly dynamic network. We do not explore this issue in detail, however, by managing the resources based on the network communication channel configuration, we can envision that the same conclusions can be foreseen. Without an integrated technology, ITS-related services are going to fail due to the unreliable network performance, and likely won't have the impact it is being expected to have.

\section{Conclusion}

ITS is progressing towards a system where vehicles have communication capabilities to maximise users experience and safety on the road and, at the same time, enhance its overall performance through the next generation of mobile networks, or 5G. The central challenge of this scenario is to develop a framework that is capable of concealing ITS requirements with 5G solutions by adapting the network to the dynamic network behaviour found in ITS. In this paper, the Cognition-enabled network management for 5G V2X Communication (CogITS) architecture is presented. A machine learning based technique extracts knowledge of the network patterns, aggregates it into a context, and coordinates actions among network controllers, enabling the cognitive network management. We presented a preliminary proof-of-concept showing how the coordination among RSU by the management solution can decrease latency by adapting the network to accommodate its requirements with its infrastructure. The future direction of this technology will be based on the combination of resources and service management to improve overall system performance in a cross-layer based approach.

\section{References}

1 C. Han, M. Dianati, Y. Cao, F. Mccullough, and A Mouzakitis, "Adaptive network segmentation and channel allocation in large-scale v2x communication networks," IEEE Transactions on Communications, vol. 67, no. 1, pp. 405-416, 2018.

2 Z. He, J. Cao, and X. Liu, "SDVN: Enabling Rapid Network Innovation for Heterogeneous Vehicular Communication," IEEE network, vol. 30, no. 4, pp. 10-15, 2016
3 M. T. Barros and R. Mullins, "Cognitive Network Management for 5G," in 5GPPP White Papers, 2017.

4 B. Rong, M. Dianati, L. Zhou, G. K. Karagiannidis, and C. Wang, " $5 \mathrm{~g}$ mmwave small cell networks: Architecture, self-organization, and management," IEEE Wireless Communications, vol. 25, no. 4, pp. 8-9, 2018

5 K. Zheng, L. Hou, H. Meng, Q. Zheng, N. Lu, and L. Lei, "Soft-defined heterogeneous vehicular network: Architecture and challenges," IEEE Network, vol. 30 , no. 4 , pp. $72-80,2016$

6 J. Wan, D. Zhang, S. Zhao, L. Yang, and J. Lloret, "Context-aware vehicular cyberphysical systems with cloud support: architecture, challenges, and solutions," IEEE Communications Magazine, vol. 52, no. 8, pp. 106-113, 2014.

7 H. Arregui, A. Mujika, E. Loyo, G. Velez, M. T. Barros, and O. Otaegui, "Short-term vehicle traffic prediction for terahertz line-of-sight estimation and optimization in small cells," IEEE Access, 2019.

8 Z. Ning, J. Huang, and X. Wang, "Vehicular fog computing: Enabling real-time traffic management for smart cities," IEEE Wireless Communications, vol. 26 , no. 1 , pp. 87-93, 2019.

9 N. Cheng, F. Lyu, J. Chen, W. Xu, H. Zhou, S. Zhang, and X. Shen, "Big data driven vehicular networks," IEEE Network, no. 99, pp. 1-8, 2018.

10 K. Wang, H. Yin, W. Quan, and G. Min, "Enabling collaborative edge computing for software defined vehicular networks," IEEE Network, no. 99, pp. 1-6, 2018.

11 X. Chen, N. Thomas, T. Zhan, and J. Ding, "A hybrid task scheduling scheme for heterogeneous vehicular edge systems," IEEE Access, vol. 7, pp. 117088-117099, 2019.

12 G. ETSI, "Network functions virtualisation (nfv): Architectural framework," ETSI GS NFV, vol. 2, no. 2, p. V1, 2013.

13 M. Chiosi, D. Clarke, P. Willis, A. Reid, J. Feger, M. Bugenhagen, W. Khan, M. Fargano, C. Cui, H. Deng, et al., "Network functions virtualisation: An introduction, benefits, enablers, challenges and call for action," in SDN and OpenFlow World Congress, pp. 22-24, 2012.

14 M. T. Barros, R. Mullins, and S. Balasubramaniam, "Integrated terahertz communication with reflectors for $5 \mathrm{~g}$ small-cell networks," IEEE Transactions on Vehicular Technology, vol. 66, no. 7, pp. 5647-5657, 2017.

15 D.-T. Phan-Huy, M. Sternad, and T. Svensson, "Making $5 \mathrm{~g}$ adaptive antennas work for very fast moving vehicles," IEEE Intelligent Transportation Systems Magazine, vol. 7, no. 2, pp. 71-84, 2015.

16 G. Bianchi, E. Biton, N. Blefari-Melazzi, I. Borges, L. Chiaraviglio, P. Cruz Ramos, P. Eardley, F. Fontes, M. J. McGrath, L. Natarianni, et al., "Superfluidity: a flexible functional architecture for $5 \mathrm{G}$ networks," Transactions on Emerging Telecommunications Technologies, vol. 27, no. 9, pp. 1178-1186, 2016.

17 M. T. Barros, R. Gomes, M. S. de Alencar, and A. F. da Costa, "IP traffic classifiers applied to DiffServ networks," in IEEE Symposium on Computers and Communications (ISCC), IEEE, 2013.

18 K. D. Singh, P. Rawat, and J.-M. Bonnin, "Cognitive radio for vehicular ad hoc networks (cr-vanets): approaches and challenges," EURASIP journal on wireles communications and networking, vol. 2014, no. 1, p. 49, 2014

19 H.-J. Günther, S. Kleinau, O. Trauer, and L. Wolf, "Platooning at traffic lights," in IEEE Intelligent Vehicles Symposium (IV), IEEE, 2016.

20 K. Sharma, B. Butler, B. Jennings, J. Kennedy, and R. Loomba, "Optimizing the placement of data collection services on vehicle clusters" in 2018 IEEE 29th Annual International Symposium on Personal, Indoor and Mobile Radio Communications (PIMRC), pp. 1800-1806, IEEE, 2018.

21 K. T. Bagci, S. Yilmaz, K. E. Sahin, and A. M. Tekalp, "Dynamic end-to-end service-level negotiation over multi-domain software defined networks," in 2016 IEEE Sixth International Conference on Communications and Electronics (ICCE), pp. 33-39, July 2016.

22 R. R. Fontes, S. Afzal, S. H. B. Brito, M. A. S. Santos, and C. E. Rothenberg, "Mininet-wifi: Emulating software-defined wireless networks," in 2015 11th International Conference on Network and Service Management (CNSM), pp. 384-389, Nov 2015.

23 A. De La Oliva, X. C. Pérez, A. Azcorra, A. Di Giglio, F. Cavaliere, D. Tiegelbekkers, J. Lessmann, T. Haustein, A. Mourad, and P. Iovanna, "Xhaul: toward an integrated fronthaul/backhaul architecture in 5G networks," IEEE Wireless Communications, vol. 22, no. 5, pp. 32-40, 2015. 